\title{
Hepatocellular Carcinoma screening is indicated Even After Sustained Virological Response: Moroccan University Hospital Experience-
}

\author{
Y. Cherradi ${ }^{1,3}$, R. Afifi ${ }^{1,3}$, H. Benbrahim ${ }^{1,3}$,O. H. El Malki ${ }^{2,3}$, M. Benazzouz ${ }^{1,3}$, A. Essaid E $^{1,3}$. \\ 1 Department of Hepatogastroenterology "Médecine C", Ibn Sina University Hospital, Rabat, Morocco. \\ ${ }^{2}$ Biostatistics, Clinical and Epidemiological Research Laboratory, Mohammed V ${ }^{\text {th }}$ University- Souissi, Rabat, Morocco \\ ${ }^{3}$ Medical School of Rabat, Mohammed Vth University, Rabat, Morocco.
}

Corresponding author:

Dr Younes Cherradi, MD.

Adress: Department of Hepatogastroenterology "Médecine C", Ibn Sina University Hospital, Rabat, Morocco.

Tel: 00212665659284.

E-mail: cherradiyounes@hotmail.com

Copyright () 2012- 2016. Dr Y. Cherradi. and al. This is an open access article published under Creative Commons Attribution-NonCommercial-NoDerivs 4.0 International Public License (CC BY-NC-ND). This license allows others to download the articles and share them with others as long as they credit you, but they can't change them in any way or use them commercially.

\section{Abstract}

Introduction: Hepatitis $\mathrm{C}$ is the first aetiologic agent for $\mathrm{HCC}$ in Morocco. Antiviral treatment reduces the risk of developing $\mathrm{HCC}$ in patients with chronic hepatitis $\mathrm{C}$ but few cases of $\mathrm{HCC}$ have been still reported. We aimed to define population with high risk of $\mathrm{HCC}$ occurrence, confirm the protective role of SVR and to identify predictive factors of developing HCC after SVR. We'll try to present suggestions about screening strategies (indications and interval) after antiviral therapy according to level of HCC occurrence risk.

Patients and Methods: We included all patients with chronic hepatitis $\mathrm{C}$ treated in our department from January 2002 to April 2010. We compare HCV-treated patients with no developed HCC to HCC population using khi-2 and Fisher Exact analysis.

Results: 369 patients treated for hepatitis $\mathrm{C}$ were considered, and $20 \mathrm{HCC}$ were reported. The risk of HCC occurrence was not significant according to gender and genotypes. Advanced age and severe fibrosis were significant risk factors. HCC was reported in $2.3 \%$ of sustained virological responders versus $12.5 \%$ of non responders. SVR is a significant protective factor.

Conclusion: In our series, 5\% of previously treated HCV carriers developed HCC and 2.3\% of sustained virological responders developed HCC. Achieving SVR after antiviral therapy is a protective factor. Advanced age $(>50$ y. o), severe fibrosis $(\mathrm{F}>2)$ and lack of SVR at HCV diagnosis are predictive factors of HCC development in treated patients. Regular bi-annual ultrasonography screening should be indicated necessarily in patients with advanced fibrosis stage (F3- F4) even after SVR, particularly when co-morbid conditions are associated (advanced age, NASH, diabetes mellitus,...). Screening interval in sustained virological responders with reduced fibrosis stage may be reduced to annual surveillance. Establishing guidelines about consensual strategy to survey sustained virological responders is now necessary especially with high rates of SVR and the extension of treatment indications in era of DAA drugs.

Keywords: Hepatocellular carcinoma; Screening strategy; Sustained virological response (SVR):

\section{Introduction}

Hepatitis $\mathrm{C}$ virus (HCV) is a recognized aetiologic agent that induces carcinogenesis process in the liver by generating inflammation, fibrosis, and a carcinogenic tissue microenvironment for the development of Hepatocellular Carcinoma (HCC). $\mathrm{HCC}$ is the most common malignant tumors in the world; it's also the main type of primary liver cancers and the third most common cause of cancer mortality worldwide [1]. In Morocco, according to our department register, the major risk factor for 
HCC is chronic hepatitis especially hepatitis C virus infection [2]. The risk of HCC gradually increases as liver fibrosis progresses and HCVinduced $\mathrm{HCC}$ is a rational target for cancer preventive intervention. Once cirrhosis is established, the annual incidence of $\mathrm{HCC}$ is extremely high (1\%-7\% per year) [3]. Hepatocarcinogenesis is an heterogeneous and long process and there is still much to elucidate. The combination of pegylated interferon to ribavirin (PEG/RBV) given for 24 or 48 weeks -according to genotype- has been retained for long years ago as the only consensual and standard treatment of chronic hepatitis $\mathrm{C}$ before the development of antiproteasis and anti polymerases. Recent clinical trials have reported sustained virological response (SVR) rates greater than $90 \%$ with the use of Direct-Acting Antiviral drugs (DAA)-based interferon-free regimens even in cirrhotic and hardto-treat patients [3]. Antiviral treatment limits fibrosis progression and the incidence of HCC has been shown to be reduced after antiviral therapy. However, few cases of HCC in patients with chronic hepatitis $\mathrm{C}$ are still reported even after antiviral treatment. This study investigates the risk of developing $\mathrm{HCC}$ in chronic hepatitis $\mathrm{C}$ carriers after antiviral therapy. It aims to identify population with high risk of HCC occurrence and to confirm the protective role of SVR. The major endpoint is to define predictors of developing HCC after achieving SVR. We'll try to present suggestions about screening strategies (indications and interval) after antiviral therapy according to level of HCC occurrence risk.

\section{Patients and methods}

It's a monocentric, retro-prospective and analytic study. It includes all patients with chronic hepatitis C enrolled in our department from January 2002 and April 2010, who were considered for antiviral treatment. At study onset, included patients had no HCC and they were not co-infected by hepatitis B or HIV. They were all tested for HCC before inclusion: abdominal ultrasonography was systematically performed prior to antiviral therapy. Patients with current or previous history of HCC were excluded. All participants were treated by Peg-IFN/RBV. During the follow-up, all patients benefited from ultrasonography biannually after treatment to detect suspected hepatic nodules.

Diagnosis of HCC was retained on the basis of histological confirmation when biopsy was performed or according to morphological non invasive criteria (Barcelona criteria) for cirrhotic patients where biopsy was not necessary. For each patient, we considered following metadata: age at
HCV diagnosis, gender, baseline viral loads (BVL), Hepatitis $\mathrm{C}$ genotype, necro-inflammatory activity (A) and fibrosis (F) degrees at the beginning of antiviral treatment. Histological activity and fibrosis were evaluated according to METAVIR score ( $\mathrm{F}>2$ was considered as an advanced stage of fibrosis). We considered initially all treated patients regardless of treatment outcome and we compared treated patients who didn't develop HCC at the follow-up to patients who developed HCC; we used khi-2 and Ficher-Exact analysis. In a second step, we were particularly interested in the population of patients who developed HCC even they achieved SVR in order to elucidate the risk factors of developing HCC after SVR. We compared sustained virological responders who didn't develop HCC to sustained virological responders with developed HCC in the follow-up. we used Pearson chi-square test. Patients included in this study had at least $\mathbf{0 3}$ years of regular biannual follow-up.

\section{Results:}

Three hundred sixty nine patients received antiviral treatment for hepatitis $\mathrm{C}$ in our department from January 2002 to April 2010 and were then included in our study. Time of follow up ranges between 3 and 9 years with a median of 6 years. During the regular bi-annual follow up, $20 \mathrm{HCC}$ has been diagnosed which corresponds to $5 \%$ of previously treated population. In the 20 participants who developed HCC, 12 were female $(60 \%)$ and 8 male (40\%); the mean age was 61 years old [40-72]. The mean time for HCC occurrence was $5+/-2$ years. Fifty Tree percent of patients had a HCV-genotype 1 (HCV-1) and 47\% had HCV-genotype 2 (HCV$2)$. Ninety five percent presented severe fibrosis at the beginning of treatment (Table I). SVR was achieved in 259 patients $(70 \%)$. In patients with SVR, only 06 of 259 developed HCC (2. 3\%) fig. 1 .

Considering all treated population, the comparison of HCC occurrence rate in patients who didn't achieve SVR to sustained virological responders shows significant results: HCC was reported in $2.3 \%$ of sustained virological responders vs. $12.5 \%$ of non-SVR patients $(\mathrm{p}=0.004)$ (Fig. 1) which confirms that SVR reduces significantly the risk of developing HCC. The risk of HCC development was not significant according to patients gender and Hepatitis $\mathrm{C}$ genotypes (respectively $\mathrm{p}=0.63$ and $\mathrm{p}=0.87$ ). Advanced age at diagnosis and severe fibrosis were significant risk factors (respectively $\mathrm{p}=0.003$ and $\mathrm{p}=0.0001$ ) (Table II).

Of the 20 patients who developed HCC, six were previously sustained virological responders. They 
Journal of Medical and Surgical Research

Table II: predictors of developing HCC in treated HCV carriers

\begin{tabular}{llrl} 
& & $\mathrm{n}(\%)$ & $\mathrm{P}$ \\
\hline Gender & Male & $148(40 \%)$ & $\mathrm{p}=0.63$ \\
Age of initial diagnosis of HCV & Female & $221(60 \%)$ & \\
\cline { 2 - 3 } Genotype & $>60$ y.o & $277(75 \%)$ & $\mathrm{p}=0.003$ \\
& $\leq 60 \mathrm{y} \cdot \mathrm{o}$ & $92(25 \%)$ & \\
\cline { 2 - 3 } Initial fibrosis & Genotype 1 & $196(53 \%)$ & $\mathrm{p}=0.87$ \\
& Genotype 2 & $173(47 \%)$ & \\
\cline { 2 - 3 } Treatment Response & F1-F2 & $18(5 \%)$ & $\mathrm{p}=0.0001$ \\
& F3-F4 & $351(95 \%)$ & \\
\cline { 2 - 3 } & SVR achieved & $118(32 \%)$ & $\mathrm{p}=0.004$ \\
& non achieved SVR & $251(68 \%)$ & \\
\hline
\end{tabular}

Table III: Advanced fibrosis is the only significant risk factor of HCC development after achieving SVR.

\begin{tabular}{|c|c|c|c|c|c|}
\hline & & $\begin{array}{l}\text { Patients with developed } \\
\text { HCC }\end{array}$ & & Patients with no HCC & $\mathrm{P}$ \\
\hline \multirow[t]{2}{*}{ Gender } & Male & $3.3 \%$ & Vs. & $96.7 \%$ & \multirow{2}{*}{$\mathrm{p}=1$} \\
\hline & Female & $3.4 \%$ & Vs. & $96.6 \%$ & \\
\hline \multirow[t]{2}{*}{ Age of diagnosis } & Age $\leq 55$ & $1 \%$ & Vs. & $99 \%$ & \multirow{2}{*}{$\mathrm{p}=0.1$} \\
\hline & Age $>55$ & $5.7 \%$ & Vs. & $94.3 \%$ & \\
\hline \multirow[t]{2}{*}{ HCV Genotypes } & 1 & $1.3 \%$ & Vs. & $98.7 \%$ & \multirow[b]{2}{*}{$\mathrm{p}=0.4$} \\
\hline & 2 & $4.7 \%$ & Vs. & $95.3 \%$ & \\
\hline \multirow[t]{2}{*}{ Baseline viral loads (BVL) } & High BVL & $3 \%$ & Vs. & $97 \%$ & \multirow{2}{*}{$\mathrm{p}=0.57$} \\
\hline & Low BVL & $1.1 \%$ & Vs. & $98.9 \%$ & \\
\hline \multirow[t]{2}{*}{ Necro inflammatory activity } & $\mathrm{A} \leq 2$ & $3.2 \%$ & Vs. & $96.8 \%$ & \multirow[b]{2}{*}{$\mathrm{p}=0.4$} \\
\hline & $\mathrm{A}>2$ & $6.3 \%$ & Vs. & $93.7 \%$ & \\
\hline \multirow[t]{2}{*}{$\underline{\text { Fibrosis }}^{* * * *}$} & $\mathrm{~F} \leq 2$ & $0 \%$ & Vs. & $100 \%$ & \multirow[b]{2}{*}{$\mathrm{p}=0.01$} \\
\hline & $\mathrm{F}>2$ & $8.8 \%$ & Vs. & $91.2 \%$ & \\
\hline
\end{tabular}

\section{Discussion}

Hepatitis $\mathrm{C}$ is a real public health problem worldwide. It's an oncogenic virus with a particular tropism for the liver and a well-known etiology of HCC. In Morocco, according to recently conducted meta-analysis of the epidemiology of Hepatitis $\mathrm{C}$ in the Maghreb region, the National prevalence of Hepatitis $\mathrm{C}$ in general population was estimated to $0.8 \%$ (95\% CI: 0.5-1.2) [4]. In a National crosssectional survey among 41269 participants, the overall prevalence of $\mathrm{HCV}$ infection in the general population was found to be $1.58 \%$ [5]. In other hand, prevalence was estimated to $74 \%$ and $44 \%$ in individuals with chronic or acute hepatitis $[4,6]$; a Moroccan study interested in a selected population of patients with $\mathrm{HCC}$ reported a prevalence rate of $57 \%[4,7]$.

Each year, HCC is diagnosed in more than half a million people worldwide, including approximately 20.000 new cases in the United States [8]. Risk factors for HCC include infection with hepatitis B or $\mathrm{C}$, alcoholic liver disease, nonalcoholic fatty liver disease and exposure to environmental toxins especially aflatoxine. In the North Africa, despite of the high prevalence of HBV infection, HCC development seems to be more associated with hepatitis $\mathrm{C}$ than hepatitis B [9]. It's known in the HVC infection's natural history that $20 \%$ of infected patients will develop severe chronic hepatitis $\mathrm{C}$ and cirrhosis which is considered as a precancerous condition predisposing to $\mathrm{HCC}[10,11]$. The HCVinduced HCC development is a multi-step process that creates persistent chronic hepatic inflammation, progressive fibrogenesis in the hepatocysts, initiation of neoplastic clones accompanied by irreversible somatic genetic/epigenetic alterations, and progression of the malignant clones in a carcinogenic tissue microenvironment [3]. This process could take $20-40$ years.

The great advantage in the management of chronic hepatitis C compared to hepatitis B is that Hepatitis $\mathrm{C}$ virus can be completely eradicated after treatment. Indeed, antiviral therapy of chronic hepatitis $\mathrm{C}$ has been developed rapidly and, in thirty years, SVR rates increased from $6 \%$ to up to $90 \%$. Actually, after introducing DAA drugs, it's established definitively that Hepatitis $\mathrm{C}$ is a curable infection. 
The new drugs, that can be combined to provide short and well tolerated all oral regimens, allows high rates of SVR for all HCV genotypes even in patients previously considered "difficult-to-treat" and in patients with advanced cirrhosis that in the past were contraindicated to antiviral treatment. [12] Our study was interested in the outcome of $\mathrm{HCV}$ carriers who benefited of antiviral therapy; in our series, antiviral treatment was the association PegIFN/RBV which has been retained for long time as standard of care in $\mathrm{HCV}$-chronically-infected patient. The major assessment was that HCC may occur in treated patients even if SVR has been successfully achieved.

The predictors of HCC occurrence in treated HCV carriers were advanced age (> 50 years old), severe fibrosis and lack of SVR. Our study suggests that older patients -over 50 years old- were a risk factor population for HCC occurrence after antiviral treatment. This was reported by Nihon Rinsho who showed that the risk for developing HCC depends on age and is more important after 65 years-old [13]. Furthermore, it was demonstrated that the benefit of HCV eradication on HCC prevention was less significant in older patients than in younger [13]. Our patients with severe fibrosis were the second risk factor population; it's established in the literature data that the risk of HCC gradually increases as liver fibrosis progresses. Once cirrhosis is established, the annual incidence of $\mathrm{HCC}$ is extremely high (1-7\% per year), although $\mathrm{HCC}$ rarely develops in less fibrotic livers [3, 14]. Finally, the protective role of SVR [3] has been demonstrated again in our series. The lack of SVR exposes significantly to HCC development. Continuing biannual regular screening of $\mathrm{HCC}$ in non-responders patients is the rational strategy. Some authors suggest that the lack of surveillance after SVR was obviously associated with more advanced HCC at detection, resulting in poor prognosis. In addition, there is a difference in the severity of HCC at diagnosis according to the surveillance interval [3].

It's certain that achieving SVR improves the prognosis of patients and limits fibrosis progression which reduces the risk of HCC development. This preventive effect may be explained by the antiinflammatory effect of stopping necro-inflammatory mechanisms responsible of progression of fibrosis in the previously infected liver. However, HCV eradication does not abolish definitely the risk of HCC [15]. In our series, 2.3\% of sustained virological responders developed HCC: the previous carcinogenic effects of $\mathrm{HCV}$ proteins may continue to create a cellular tissue microenvironment that serves to tumor evolution even after virus eradication. This demonstrates that screening is still indicated even after SVR! This founding led to the big question of screening strategy (surveillance interval, surveillance duration and high risk population) after SVR. If we established that screening is indicated even after SVR, should we process with bi-annual ultrasonography screening even if fibrosis stage is reduced (F1- F2)?

Actually, regression of cirrhosis is now a recognized concept $[16,17]$. Liver fibrosis has been defined as a dynamic and potentially bidirectional process and the spontaneous regression of fibrosis has been demonstrated in both animal models of hepatic fibrosis and human trials in which the responsible agent of chronic hepatitis has been successfully controlled, like SVR condition [16]. In the coming decades, regression of cirrhosis should become the secondary reasonable goal after inactivation of viral hepatitis $\mathrm{C}$ [17]. HCV infection is associated with a 15- to 20-fold increase in risk for HCC compared with HCV-negative subjects in cross-sectional and case-control studies [18]. This could suggest that patients with reduced fibrosis at HCV diagnosis, who achieved SVR after antiviral therapy, present a lowest risk for developing HCC. The interval of regular screening in those patients could be reduced to annual surveillance only.

One of our study's endpoints was to define risk factors of HCC occurrence in sustained virological responders to define the profile of patients who should be carefully and closely considered for HCC screening after SVR. In our series, all the sustained virological responders who developed HCC had an advanced fibrosis stage ( $\mathrm{F}>2$ according to Metavir Score) and it has been shown statistically that this condition is the only significant risk factor for developing HCC after achieving SVR.

Establishing guidelines about consensual strategy to survey sustained virological responders is necessary. If it's clearly estabilished that regular bi-annual screening is indicated in patients with advanced fibrosis stage even more when co-morbid conditions are associated (such as older patients, diabetes mellitus, nonalcoholic fatty liver disease, alcoholism...), it becomes strongly important to stay about the surveillance strategy in the other patients categories. We are now in the era of anti-viral drugs associated to higher SVR rates; also, HCV therapy is indicated today in patients with reduced fibrosis as a major element of the global vision for HCC prevention and $\mathrm{HCV}$ eradication worldwide.

\section{Conclusion}

In our series, $5 \%$ of previously treated $\mathrm{HCV}$ carriers developed HCC and $2.3 \%$ of sustained virological responders developed. Our results confirm the evidence that achieving SVR after antiviral therapy 
is a protective factor which decreases significantly the incidence of $\mathrm{HCC}$ in patients with $\mathrm{HCV}$. Advanced age (> 50 y. o) severe fibrosis $(\mathrm{F}>2)$ and lack of SVR at HCV diagnosis are predictive factors of HCC development in treated patients. According to our real-life experience, regular bi-annual ultrasonography screening should be indicated necessarily in patients with advanced fibrosis stage (F3- F4) even after SVR, particularly when comorbid conditions are associated (advanced age, $\mathrm{NASH}$, diabetes mellitus,...). Screening interval in sustained virological responders with reduced fibrosis stage may be reduced to annual surveillance. Establishing guidelines about consensual strategy to survey sustained virological responders is now necessary especially with high rates of SVR and the extension of treatment indications in era of DAA drugs.

\section{References}

1- Epidemiology, natural history and pathogenesis of hepatocellular carcinoma. Maillard E. Cancer Radiother. 2011 Feb; 15 (1):3-6.

2- Bahri O, Ezzikouri S, Ben Alaya-Bouafif N, Iguer F, A. E. Essaid A. E, Mestir H, and all. First multicenter study for risk factors for hepatocellular carcinoma development in North Africa, World J Hepatol 2011 January 27; 3(1): 24-30

3- Hoshida Y, Fuchs BC, Bardeesy N, Baumert TF, Chung RT. Pathogenesis and prevention of hepatitis $\mathrm{C}$ virus-induced hepatocellular carcinoma. J Hepatol. 2014 November; 61(1 Suppl): S79-S90. doi: 10.1016/j.jhep.2014.07.010

4- Fadlalla FA, Mohamoud YA, Mumtaz GR, AbuRaddad LJ. The Epidemiology of Hepatitis C Virus in the Maghreb Region: Systematic Review and Meta-Analyses. Thorne C, ed. PLoS ONE. 2015;10(3):e0121873. doi:10.1371/journal. pone.0121873.

5- Baha W, Foullous A, Dersi N, Paluku T, El Alaoui $\mathrm{K}$, Nourichafi N, and all. Prevalence and risk factors of hepatitis $\mathrm{B}$ and $\mathrm{C}$ virus infections among the general population and blood donors in Morocco. BMC Public Health BMC series 2013. DOI: $10.1186 / 1471-2458-13-50$

6- Rioche M, Himmich H, Cherkaoui A, Mourid A, Dubreuil P, Zahraoui $\mathrm{M}$, and al. High incidence of sporadic non-A, non-B hepatitis in Morocco: epidemiologic study. Forte incidence des hepatites non-A, non-B sporadiques au Maroc: etude epidemiologique. Bulletin de la Societe de pathologie exotique (1990). 1991;84(2):117-27.

7- Ezzikouri S, El Feydi AE, Afifi R, El Kihal L, Benazzouz M, Hassar M, et al. MDM2 SNP309T>G polymorphism and risk of hepatocellular carcinoma: a case-control analysis in a Moroccan population.Cancer detection and prevention. 2009; 32(5-6):380-5. Epub 2009/02/24. doi: 10.1016/j.cdp.2009.01.003
8- Hashem B. El-Serag. N Engl J Med 2011; 365:11181127September $22, \quad 2011 \quad$ DOI: 10.1056/NEJMra1001683

9- Bahri O, Ezzikouri S, Ben Alaya-Bouafif N, Iguer F, A. Essaid A. E, Mestiri H, and all. Factors for hepatocellular carcinoma development in the North Africa. Arab Journal of Gastroenterology, Volume 10, Issue 2, June 2009, Page AB15 doi:10.1016/j.ajg.2009.07.023

10- Younès Cherradi, Rajaa Afifi, Hanaa Benbrahim, et al., "Predictors of Developing Hepatocellular Carcinoma in Treated HCV-Carriers in Morocco according to University Hospital Experience," ISRN Hepatology, vol. 2013, Article ID 438306, 3 pages, 2013. doi: $10.1155 / 2013 / 438306$

11- Michielsen PP, Francque SM, van Dongen JL. Viral hepatitis and hepatocellular carcinoma. World J Surg Oncol. 2005 May 20; 3: 27.

12- Minerva, De Nicola S1, Colombo M. The eradication of HCV. Gastroenterol Dietol. 2015 Oct 7.

13- Asahina Y, Nihon R. Anti-HCV treatment for older patients. $2015 \mathrm{Feb} ; 73(2): 292-8$.

14- -Serag, Hashem B. "Epidemiology of Viral Hepatitis and Hepatocellular Carcinoma. Gastroenterology 142.6 (2012): 1264-1273.e1. PMC. Web. 29 May 2016.

15- Toyoda H, Tada T, Tsuji K, Hiraoka A, Tachi Y, Itobayashi E, and all. Characteristics and Prognosis of Hepatocellular Carcinoma Detected in Patients with Chronic Hepatitis C after the Eradication of Hepatitis C Virus: a Multicenter Study from Japan. Hepatol Res. 2015 Oct 27. doi: 10.1111/hepr.12613.

16- Pellicoro A, Prakash Ramachandran P. and John P Iredale J. P. Reversibility of liver fibrosis. Fibrogenesis \& Tissue Repair 20125 (Suppl 1):S26 DOI: 10.1186/1755-1536-5-S1-S26

17- Farrell GC, Chan HL, Yuen MF, Amarapurkar DN, Chutaputti A, and al. Prevention of hepatocellular carcinoma in the Asia-Pacific region: consensus statements. Asia-Pacific Working Party on Prevention of Hepatocellular Carcinoma. J Gastroenterol Hepatol. 2010 Apr; 25(4):657-63.

18- Vallet-Pichard A, Pol S, Mallet V. Assessment and reversibility of liver fibrosis in viral hepatitis. Rev Prat. 2011 Jan; 61(1):39-43. 